\title{
TIMING-MISMATCH ANALYSIS IN HIGH-SPEED ANALOG FRONT-END WITH NONUNIFORMLY HOLDING OUTPUT
}

\author{
Sai-Weng Sin ${ }^{l}$, Seng-Pan $U^{l}$, R.P.Martins ${ }^{1}$, J.E.Franca ${ }^{2}$ \\ 1 - Faculty of Science and Technology \\ University of Macau, Av. Padre Tomás Pereira S.J., Macau SAR, China \\ E-mail - benspu@umac.mo \\ (1' - on leave from IST, E-mail - rmartins@umac.mo) \\ 2 - Instituto Superior Técnico, \\ Av. Rovisco Pais, 1, 1096 Lisboa Codex, Portugal \\ E-mail - jfranca@chipidea.com
}

\begin{abstract}
This paper will present a practical and complete analysis of timing-mismatch effects for high-speed Analog Front-End (AFE) systems with the inherent nonuniformly holding outputs. The analysis reveals first the relationship with traditional impulsesampled timing-mismatch effects and then its closed-form expressions of the signal-to-noise-ratio (SNR), in terms of the number of channels, signal frequency, and jitter errors. Both timing errors imposed by random clock-jitter and fixed periodic clockskew will be analyzed. Practical analysis examples for a very highspeed data-converter as well as a AFE filtering will be addressed to illustrate the effectiveness of the derived formula.
\end{abstract}

\section{INTRODUCTION}

The rapid evolution of electronic instruments and data communication requires high-speed data conversion channels as well as signal processing units. While the current CMOS process technology limits the achievable speed of the electronics devices, time-interleaved (TI) architectures are one of the most effective ways to boost the maximum speed of the analog interface [1-4], e.g. time-interleaved ADC and DAC for high-speed analog front-end, as shown in Fig.1 [3]. However, due to the nature of TI structures, timing-mismatch of sampling clock phases among different paths can greatly degrade the system performance [5-8], and such jitter noise become much more significant in high-speed systems. Such timing-mismatches have generally two different aspects: the first is caused by the random sampling jitter which results in an increased noise floor over all frequencies. The second one is due to the unmatched but fixed periodic timing-skew among different channels, resulting in the image side-bands appearing in frequency locations which are multiple of $f_{\mathrm{s}} / M$, as shown in Fig. 2, where $f_{\mathrm{s}}$ is the sampling frequency, $M$ is the period of timing-skew, and $A_{k}$ is the weight of the modulation sidebands.

If the Input signal is sampled by the system with Nonuniform time-interval then played out or represented in the discrete samples at the Output at Uniform time instances, namely, IN-OU process, which is typical in the analog to digital conversion path (timingmismatch only happens in input signal sampling), e.g. TI ADCs [1] or multirate sampled-data decimators [2]; while if the Input signal is sampled by the system with Uniformly spaced time-interval then played out the samples at the Output Nonuniformly, then the system can be referred as IU-ON process, that is the typical case in digital to analog conversion path (timing-mismatch only happens in output signal holding), e.g. TI DACs [3] or multirate sampled-data interpolators [4]. The signal spectra for these two kinds of processes with Impulse-Sampled (IS) sequence form have been

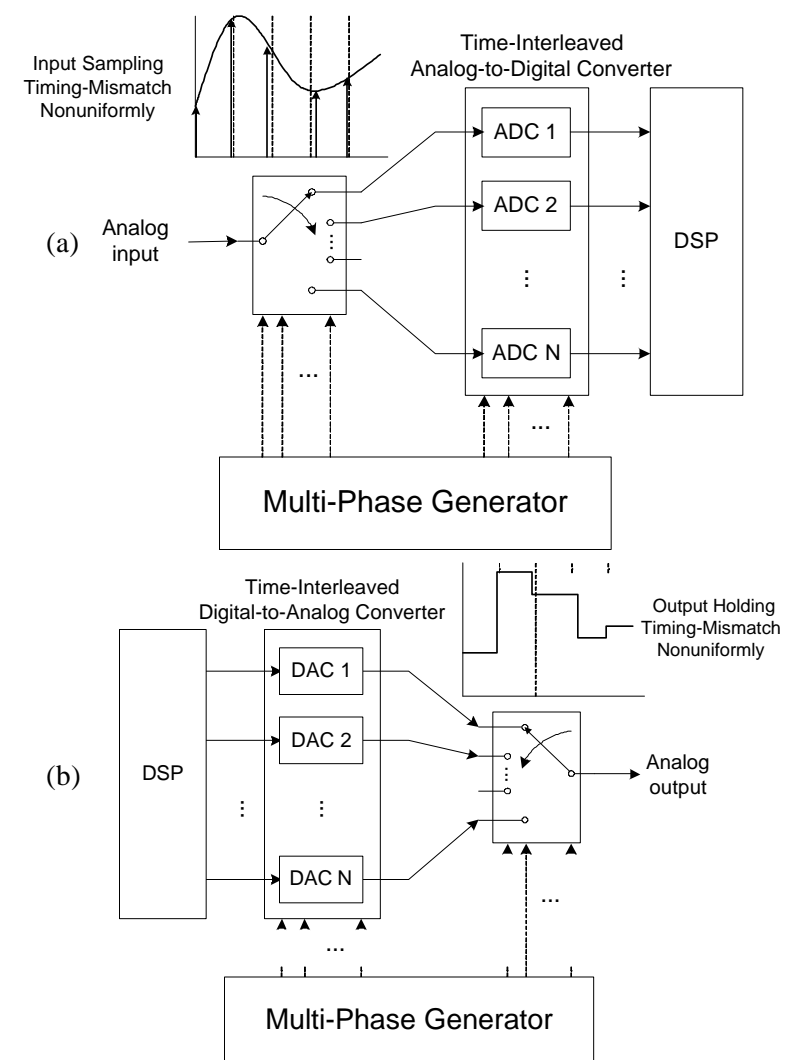

Fig. 1: General time-interleaved data converter structure:

(a) Analog to Digital (b) Digital to Analog Conversion path

analyzed in [5] and [9], respectively. However in practice, the real output signals are always in Sample-and-Hold ( $\mathrm{SH}$ ) or holding nature in IU-ON process. Especially, such extra holding effect is not simply $\sin (x) / x$ shaped version of the original IS-version spectra due to the effect of output nonuniformly holding waveform as shown in Fig.3. Therefore, the analysis of IU-ON(IS) [9] is not well suited for IU-ON(SH). This paper will present a complete description of output signal spectrum of $\mathrm{IU}-\mathrm{ON}(\mathrm{SH})$ process. Besides of this introductory part, a description of the spectra representation for IU-ON(SH) process will be given in part 2, and an interesting spectra correlation between it and IN-OU(IS) will also be described in that section. Second, the closed-form expression of the output signal-to-noise-ratio (SNR) of IU-ON(SH) system will be derived in part 3 . In part 4 , two practical examples will be illustrated to show the effectiveness of the derived formula, and finally the conclusion is drawn in part 5. 


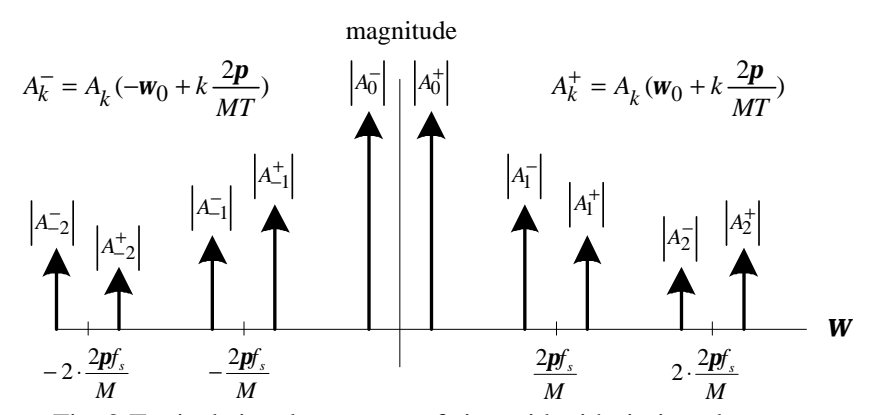

Fig. 2 Typical signal spectrum of sinusoid with timing-skew

\section{CORRELATED SPECTRA BETWEEN IN-OU(IS) \& IU-ON(SH)}

Let us first consider the IN-OU(IS) system, which ideally plays out the nonuniform input samples at uniformly spaced time intervals with impulse-sampled output nature. The output spectra of IN-OU(IS) can be represented as follows [5]:

$Y(\omega)=\frac{1}{T} \sum_{k=-\infty}^{\infty} A_{1 k_{-} I S}(\omega) \cdot X\left(\omega-k \frac{2 \pi}{M T}\right)$

where

$A_{1 k_{-} I S}(\omega)=\frac{1}{M} \sum_{m=0}^{M-1}\left(e^{j \omega r_{m} T} e^{-j k r_{m} \frac{2 \pi}{M}}\right)^{-j k m \frac{2 \pi}{M}}$

and $T=1 / f_{s}$ is the nominal sampling period and $r_{m}$ is a normalized periodic skew timing sequence with period $M(M$ is usually equal to TI path number $N$ ), measured in percentage of $T$.

Also consider the IU-ON(SH) system that ideally plays out the uniform input samples at nonuniformly spaced time intervals with holding nature. The output spectra of $\mathrm{IU}-\mathrm{ON}(\mathrm{SH})$ can be represented as (1) with different $A_{k}$ [8]:

$$
\begin{aligned}
& A_{2 k_{-} S H}(\omega)=\frac{1}{M} \sum_{m=0}^{M-1} H_{m}(\omega) e^{-j \omega r_{m} T} e^{-j k m \frac{2 \pi}{M}} \\
& H_{m}(\omega)=\frac{2 \sin \left(\omega\left(1+r_{m+1}-r_{m}\right) T / 2\right)}{\omega} e^{-j \omega\left(1+r_{m+1}-r_{m}\right) T / 2}
\end{aligned}
$$

The plots of spectra for IN-OU and IU-ON systems with both IS and SH output are shown in Fig. 4 (with normalized frequency $a=f_{o} / f_{s}=0.1, M=6$, and standard deviation of $r_{m}$ equals to $0.16 \%$ ). From the figure we can see that the IN-OU(SH) is a standard $\sin (x) / x$ shaped version of IN-OU(IS), while the situation in IU-ON

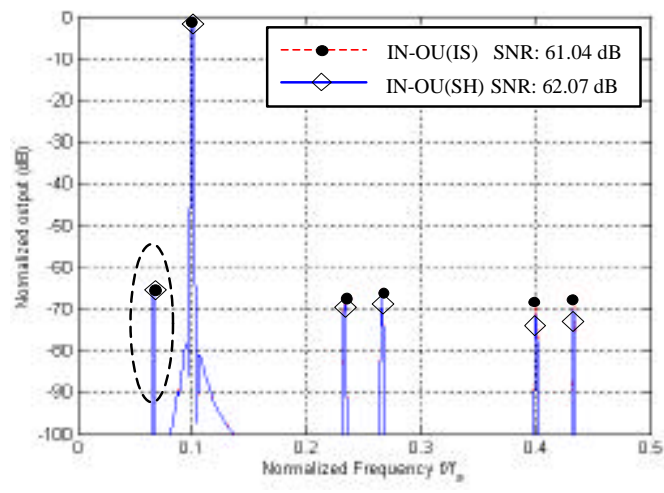

(a)

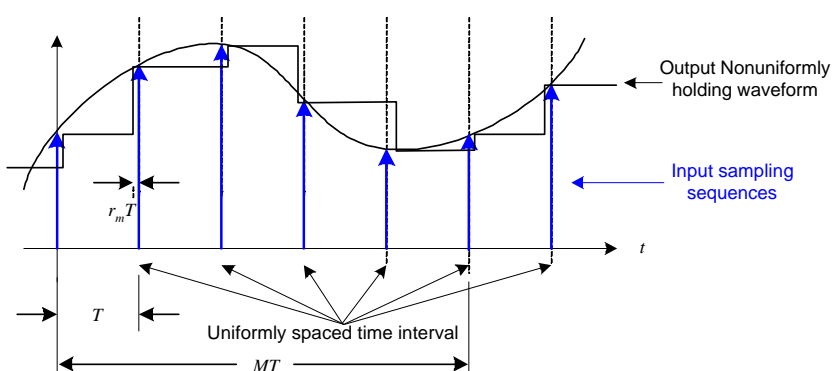

Fig.3 Input and output waveform for equivalent $\mathrm{IU}-\mathrm{ON}(\mathrm{SH})$ process

system is different (e.g. compare the circled part in figure for two cases) due to the nonuniform holding function as described in (2b).

Although from (1a), (2a) and (2b) the spectra of IN-OU(IS) and IU-ON(SH) systems are different, when with assumption of $2 p f_{o} r_{m} T<<1$, they will have identical normalized output spectra representation as shown in Fig. 4. The following proves this special correlation.

For a real input sinusoidal signal with frequency $?{ }_{0}=2 p f_{o}$, the typical output spectra of both systems can be represented as shown in Fig. 2. In the figure, $A_{k}$ represents the weighted terms of the modulated sidebands, which is equals to (1a) or (2a), and ${A_{k}}^{+}$is the terms generated by the positive frequency components of the original sine-wave, while $A_{k}^{-}$is produced by corresponding negative part. For real signal, $\left|A_{k}^{+}\right|=\left|A_{-k}^{-}\right|$, so that we can consider only $A_{k}^{+}$from $k=-\infty$ to $+\infty$, where the sidebands are located at frequency of $\omega=\omega_{o}+k(2 \pi) /(M T)$.

For the IN-OU(IS) system as described by (1a), substituting $\omega=\omega_{o}+k(2 \pi) /(M T)$ into (1a) yields

$A_{1 k_{-} I S}\left(\omega_{o}+k \frac{2 \pi}{M T}\right)=\frac{1}{M} \sum_{m=0}^{M-1}\left(e^{j \omega_{o} r_{m} T}\right) e^{-j k m \frac{2 \pi}{M}}$

Assuming $\omega_{o} r_{m} T=2 p f_{o} r_{m} T<<1$, the magnitude of sidebands components can be expressed as:

$$
\begin{aligned}
\left|A_{1 k_{-} I S}\left(\omega_{o}+k \frac{2 \pi}{M T}\right)\right| \approx \mid \frac{1}{M} \sum_{m=0}^{M-1}\left(1+j \omega_{o} r_{m} T\right) e^{-j k m \frac{2 \pi}{M} \mid} \\
= \begin{cases}\frac{\omega_{o} T}{M}\left|\sum_{m=0}^{M-1} r_{m} e^{-j k m \frac{2 \pi}{M}}\right| & \text { for } k \neq 0, \pm M, \ldots \\
1+j \omega_{o} T\left(\frac{1}{M} \sum_{m=0}^{M-1} r_{m}\right) \mid \approx 1 & \text { for } k=0, \pm M, \ldots\end{cases}
\end{aligned}
$$

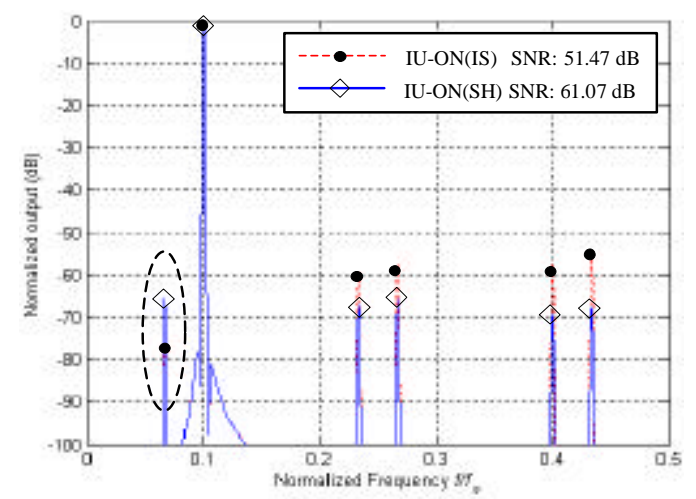

(b)

Fig. 4 FFT spectra of output sinusoid with normalized frequency of $a=0.1$ for (a) IN-OU and (b) IU-ON processes with both IS and SH output $\left(M=6, s_{r m}=0.16 \%\right)$ 
For IU-ON(SH) system, the weighted terms of the system are described in (2a) \& (2b). Since $r_{m}$ and $e^{-j k m \frac{2 \pi}{M}}$ are periodic with period $m=M$, after simplification of (2a) and (2b) with $\omega$ $=\omega_{o}+k(2 \pi) /(M T),(2 \mathrm{a})$ is given by

$A_{2 k_{-} S H}\left(\omega_{0}+k \frac{2 \pi}{M T}\right)=\frac{2 \sin \left(\omega_{0} T / 2\right)}{\left(\omega_{0}+k \frac{2 \pi}{M T}\right) M} e^{-j \omega_{0} T / 2} \sum_{m=0}^{M-1} e^{-j \omega_{0} r_{m} T} e^{-j k r_{m} \frac{2 \pi}{M}} e^{-j k m \frac{2 \pi}{M}}$

By expanding the term $e^{-j \omega_{0} r_{m} T} e^{-j k r_{m} \frac{2 \pi}{M}}$ into Taylor series $\left(r_{m} \ll<1\right)$ and taking the magnitude, (6) yields

$\mid A_{2 k_{-} S H}\left(\omega_{o}+k \frac{2 \pi}{M T}\right)= \begin{cases}\frac{2 T}{M} \sin \left(\omega_{o} T / 2\right)\left|\sum_{m=0}^{M-1} r_{m} e^{-j k m} \frac{2 \pi}{M}\right| & \text { for } k \neq 0, \pm M, \cdots \\ \frac{2}{\omega_{o}} \sin \left(\omega_{o} T / 2\right) & \text { for } k=0, \pm M, \cdots\end{cases}$

By normalizing the spectra representation (7) with respect to (8), the magnitude of these normalized sideband components of IU$\mathrm{ON}(\mathrm{SH})$ systems is identical to those of IN-OU(IS) systems described by (4), and this completes the prove.

\section{THE CLOSED-FORM EXPRESSION FOR SNR}

In this section, a closed-form expression for signal-to-noise ratio $(\mathrm{SNR})$ of IU-ON(SH) system is derived. This expression can be used to calculate, for example, the SNR for DAC with nonuniform output sample-and-hold nature. In the following formula derivation, we assume $\omega_{o} \neq k(2 \pi) /(M T)$, which means that the signal (and also the sidebands) is not exactly located at integer multiple of $f_{s} / M$.

The SNR can be found by the following formula [9]:

$$
S N R_{2-S H}=10 \log _{10}\left[\frac{\left|A_{20_{-} S H}\left(\omega_{o}\right)\right|^{2}}{\sum_{k=1}^{M-1}\left|A_{2 k_{-} S H}\left(\omega_{o}+k \frac{2 \pi}{M T}\right)\right|^{2}}\right] d B
$$

We can determine $\left|A_{20_{-} S H}\left(\omega_{o}\right)\right|^{2}$ as follow: Assuming $r_{\mathrm{m}}, m=$ $0,1,2, \ldots M-1$ to be $M$ independent, identically distributed (i.i.d) random variables with Gaussian distribution of zero mean and standard deviation of $s_{r m}\left(=s_{t} / T\right.$ where $s_{t}$ is the standard deviation of timing jitter with unit of sec), and the characteristic function is given by $E\left[e^{j \omega_{0} r_{m} T}\right]=e^{-\sigma_{r m}^{2} \omega_{0}^{2} T^{2} / 2}$. Substitute $k=0$ into (6) and by evaluating the expected value of $\left|A_{20 \_} S H\left(\omega_{0}\right)\right|^{2}=A_{20 \_} S H\left(\omega_{0}\right) A_{20 \_}^{*} S H\left(\omega_{0}\right)$ yields:

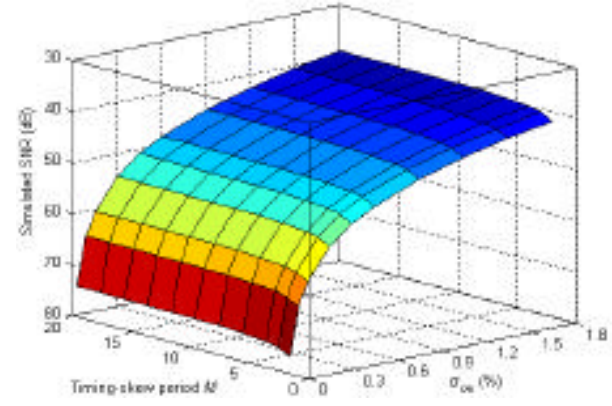

(a)

$$
\begin{aligned}
E\left[\left|A_{20 \_S H}\left(\omega_{0}\right)\right|^{2}\right] & =\frac{4}{\omega_{0}^{2} M^{2}} \sin ^{2}\left(\omega_{0} T / 2\right) \sum_{n=0}^{M-1 M-1} \sum_{m=0} E\left[e^{-j \omega_{0}\left(r_{m}-r_{n}\right) T}\right] \\
& \approx \frac{4}{\omega_{0}^{2}} \sin ^{2}\left(\omega_{0} T / 2\right)
\end{aligned}
$$

for small values of $r_{m}$ such that $2 p f_{o} r_{m} T<<1$. By similar method, we can determine the expected values of modulated sidebands components $\left|A_{2 k_{-} S H}\left(\omega_{0}+k \cdot 2 \pi /(M T)\right)\right|^{2}$ from (6):

$$
E\left[\mid A_{2 k_{-} S H}\left(\omega_{0}+k \cdot \frac{2 \pi}{M T}\right)^{2}\right] \approx \frac{4 \sigma_{r m}^{2} T^{2}}{M} \sin ^{2}\left(\omega_{0} T / 2\right)
$$

Substituting (10) \& (11) into (9) gives the SNR of IU-ON(SH) systems as

$$
S N R_{2_{-} S H}=20 \log _{10}\left(\frac{1}{2 \pi a \sigma_{r m}}\right)-10 \log _{10}\left(1-\frac{1}{M}\right)
$$

Recall that $a=f_{d} / f_{s}$ is the normalized frequency of the sinusoid. Actually, this formula is identical to the formula for IN-OU(IS) system [5], which shows that the SNR for these two systems have the same expressions for small jitter errors.

Fig. 5 shows the simulation results using MATLAB $(a=0.1)$ to illustrate the accuracy of the derived formula (12) compared with simulated SNR of IU-ON(SH) system. As shown in Fig. 5(b), the error between output SNR and that predicted by formula (12) is well below $0.1 \%$ for reasonable range of SNR, showing that (12) can well estimate the SNR of IU-ON(SH) systems.

\section{PRACTICAL APPLICATIONS}

We present here two practical examples to illustrate the theories described in the above sections in both random timing jitter and periodic timing-mismatch aspects:

1. Random jitter noise results in increasing noise floor over all frequencies: the random timing jitter is equivalent to a time-skew sequence with period $M \rightarrow \infty$. A jittered signal spectrum of $M=100$ shown in Fig. 6 presents that the modulated sidebands converge to an increased noise floor as $M$ becomes large. The calculated SNR for $s_{r m}=0.1 \%$ and $1 \%$ with $a=0.1$ are $64.04 \mathrm{~dB}$ and $44.04 \mathrm{~dB}$, respectively, comparing to the simulated $63.85 \mathrm{~dB}$ and $44.01 \mathrm{~dB}$. Moreover, the SNR increases by $20 \mathrm{~dB}$ per decade with respect to the $s_{r m}$, thus showing the consistence between the proposed theoretical prediction and practical results.

Consider an 8-bit 8Gsamples/s DAC at the transmission end of a serial-link transceiver described in [3]. To achieve 8-bit accuracy, the SNR subjected to the noise power caused by the random jitter generated by the on-chip Phase Lock-Loop must be

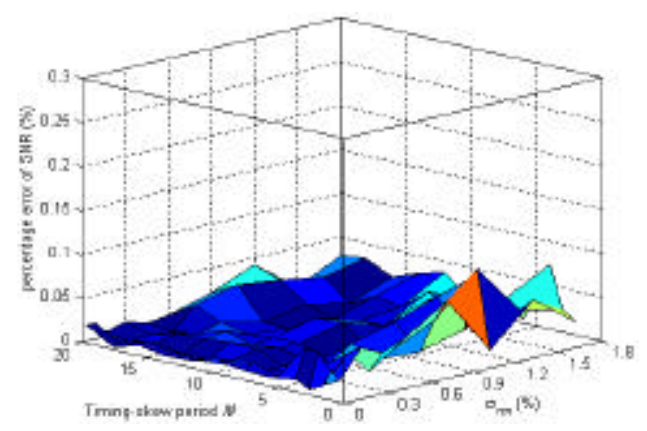

(b)

Fig. 5 (a) Simulated SNR \& (b) percentage error between the simulated and calculated SNR of IU-ON(SH) system vs. timing-skew period $M$ and the standard deviation $r_{m}$ by $10^{5}$ times Monte Carlo Simulations(normalized signal frequency $a=0.1$ ) 


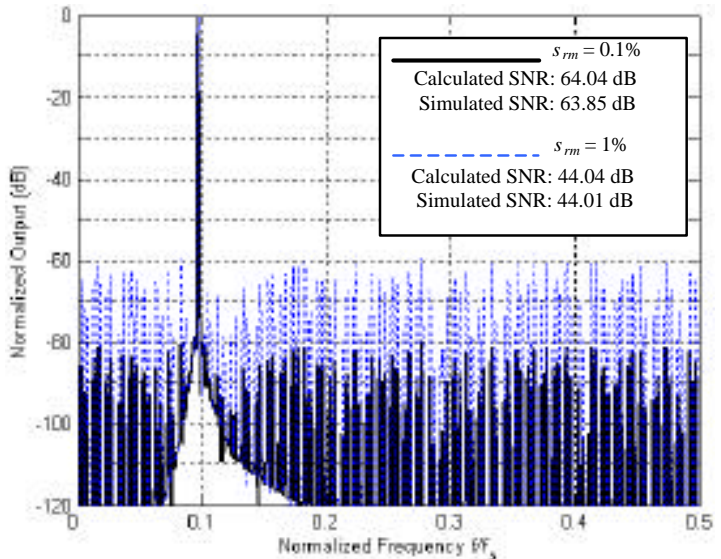

Fig. 6 FFT spectrum of output sinusoid for $\mathrm{IU}-\mathrm{ON}(\mathrm{SH})$ processes $(a=0.1 M=100)$

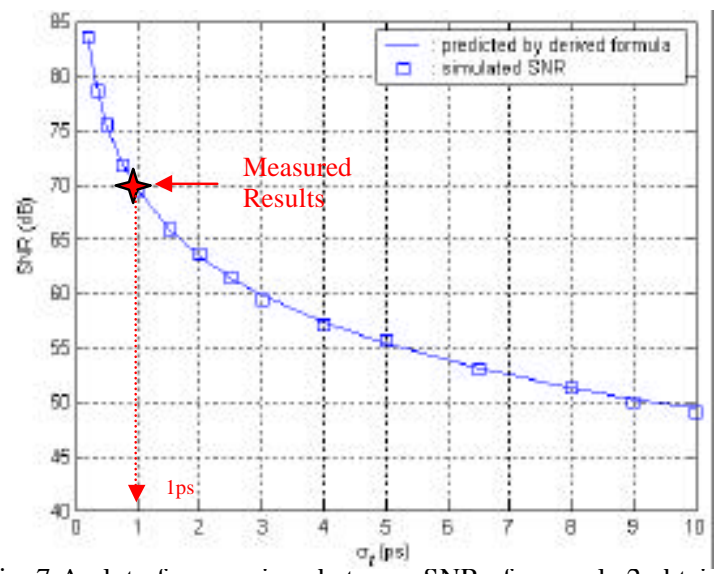

Fig. 7 A plot of comparison between SNR of example 2 obtained by MATLAB simulation and formula calculation (with $a=58 / 320=0.18$, $M=8)$

much higher than $50 \mathrm{~dB}$. Thus, at the Nyquist rate of $4 \mathrm{GHz}(a=0.5)$ the standard deviation $s_{r m}$ must be smaller than $0.1 \%$ or time jitter of 0.125 ps calculated using (12). This requirement is critical to achieve in current CMOS technology, where the measured results shows only $s_{t}$ of $2.5 \mathrm{ps}$ is realized.

2. Periodic timing skew results in modulated sidebands appearing in frequency locations of $f, M$ : This is contributed by unmatched but fixed propagation delay among the TI phase generation paths. Besides, supply variation caused by $d I / d t$ noise can destroy the matching of the rising edges of the TI phases, thus produced periodic timing skew. Consider an example of a very highfrequency (output sampling rate at $320 \mathrm{MHz}$ ) SC multirate bandpass interpolating filter in [4]. The periodic timing-skew mismatch $(M=8)$ among TI clocks is a dominating factor for the special on-chip multirate phase generator driven by an external master clock. Due to the holding nature at lower input sampling rate of analog interpolation, the timing-mismatch errors at input sampling stage are negligible, thus being equivalent to an IU$\mathrm{ON}(\mathrm{SH})$ system. From the derived formula, the standard deviation of the output jitter must be well controlled under the stringent requirement of $s_{t}<5 \mathrm{ps} \quad\left(s_{r m}<5 \mathrm{ps} \cdot 320 \mathrm{MHz}=0.16 \%\right)$ to realize SNR $>60 \mathrm{~dB}$. Simulation verifies that, the worst possible timingskew can be as large as 100ps which will completely destroy the system performance without special design and care for the multirate clock generator [8].

Fig. 7 shows the SNR from FFT simulated and calculated by derived formula vs. $s_{t}$ with signal frequency of $58 \mathrm{MHz}$ and $M=8$. This plot shows that the simulated result tracks very well the theoretical SNR curve from (12). The measured signal to the timing-jitter noise is around $70 \mathrm{~dB}[8]$, corresponding to a fixed periodic timing-skew $s_{t}$ of roughly $1 \mathrm{ps}$, which is well below the requirement of $5 \mathrm{ps}$. This proves the effectiveness of the various used design and layout techniques [8], and also shows the need of prediction of the allowed timing jitter errors during the design.

\section{CONCLUSION}

A complete analysis of timing-mismatch effects for high-speed Analog Front-End systems with practical nonuniformly holding output (IU-ON(SH)) is presented in this paper. Due to the nature that the sample-and-hold function is nonuniform in time-domain, the output spectrum is not simply a shaped version (by $\sin (x) / x$ ) of that spectra with impulse-sample output. It has been proved that the spectra of IU-ON(SH) is interestingly similar to that of IN-OU(IS) when timing-jitter is sufficiently small $\left(2 p f_{o} r_{m} T<<1\right)$. The closedform expression of the SNR for IU-ON(SH) systems has also been derived in this paper, and two practical examples are followed to illustrate the effectiveness of the derived formula. MATLAB simulation shows that the prediction accuracy of the SNR formula is within $0.1 \%$.

\section{REFERENCES}

[1] L.Sumanen,M.Waltari,K.Halonen, "A 10-bit 200MS/s CMOS parallel pipeline A/D converter," in Proc. ESSCIRC, pp.440443, Sep.2000.

[2] R.F.Neves, J.E.Franca, "A CMOS Switched-Capacitor bandpass filter with $100 \mathrm{MSample} / \mathrm{s}$ input sampling and frequency downconversion," in Proc. ESSCIRC, pp. 248-251, Sep.2000.

[3] C.-K.K.Yang, et al., "A Serial-Link Transceiver Based on 8GSamples/s A/D and D/A Converters in $0.25-\mu \mathrm{m}$ CMOS", IEEE J. Solid-State Circuits, vol. 36, no.11, pp. 1684-92, Nov.2001.

[4] Seng-Pan U, R.P.Martins, J.E.Franca, “A 2.5V, 57MHz, 15-Tap SC Bandpass Interpolating Filter with $320 \mathrm{MHz}$ Output Sampling Rate in $0.35 \mu \mathrm{m}$ CMOS," in ISSCC Dig. Tech. Papers, pp.380-382, Feb.2002.

[5] Y.C.Jenq, "Digital Spectra of Nonuniformly Sampled Signals: Fundamentals and High-Speed Waveform Digitizers," IEEE Trans. Instrum. Meas., vol. 37, no. 2, pp. 245-251, June 1988.

[6]N.Kurosawa, H.Kobayashi, K.Maruyama, et al., "Explicit Analysis of Channel Mismatch Effects in Time-Interleaved ADC Systems," IEEE Trans. CAS-I, vol.48, No.3, pp.261-271, Mar.2001.

[7]H.Kobayashi, et al., "Aperture Jitter Effects in Wideband Sampling Systems," in Proc. IMTC, pp.880-885, May 1999.

[8]Seng-Pan U, R.P.Martins, J.E.Franca, "Design and Analysis of Low Timing-Skew Clock Generation for Time-Interleaved Sampled-Data Systems," in Proc. IEEE ISCAS'2002, vol.4, pp.441-4, 2/2002.

[9]Y.C.Jenq, "Direct Digital Synthesizer with Jittered Clock," IEEE Trans. Instrum. and Meas., vol.46, No.3, pp.653-655, Jun. 1997. 